\title{
Bose-Hubbard models with staggered flux: Quantum phases, collective excitation, and tricriticality
}

\author{
Juan Yao and Shizhong Zhang \\ Department of Physics and Centre of Theoretical and Computational Physics, The University of Hong Kong, Hong Kong, China
}

(Received 6 March 2014; published 6 August 2014)

\begin{abstract}
We study the quantum phases of a Bose-Hubbard model with staggered magnetic flux in two dimensions, as was realized recently [M. Aidelsburger, M. Atala, M. Lohse, J. T. Barreiro, B. Paredes, and I. Bloch, Phys. Rev. Lett. 107, 255301 (2011)]. Within mean-field theory, we show how the structure of the condensates evolves from the weak- to the strong-coupling limit, exhibiting a tricritical point at the Mott-superfluid transition. Nontrivial topological structures (Dirac points) in the quasiparticle (hole) excitations in the Mott state are found within random phase approximation and we discuss how interaction modifies their structures. The excitation gap in the Mott state closes at different $\mathbf{k}$ points when approaching the superfluid states, which is consistent with the findings of mean-field theory.
\end{abstract}

DOI: 10.1103/PhysRevA.90.023608

PACS number(s): 03.75.Hh, 05.30.Rt

\section{INTRODUCTION}

The possibility of achieving quantum Hall [1] and other topological states [2,3] in cold-atom systems has been greatly enhanced recently with the realization of synthetic gauge fields in free space [4-9] and synthetic magnetic flux in optical lattices [10-14]. In the latter case, the flux per plaquette can approach the quantum limit, large enough to realize the Harper-Hofstadter Hamiltonian $[15,16]$ that hosts the fractal energy spectrum (Hofstadter's butterfly). With rational flux per plaquette, the lowest subband is topologically nontrivial and can give rise to quantized Hall conductance [1]. Those effects are related to the topological structure of the band structure and can be readily explored with noninteracting fermionic atoms.

The interaction effects on topological states are in general less well understood and in this regard, the cold-atom realization offers an ideal platform for addressing this issue. In optical lattices, the interaction strength can be tuned by adjusting the lattice depth and, if necessary, with Feshbach resonance. Furthermore, cold-atom systems allow the study of bosonic variants and open experimental avenues for investigating bosonic topological states, which have attracted much theoretical attention recently [17-21]. In the weak-coupling limit, Bose condensation in a uniform flux has been analyzed with Bogoliubov theory [22]. Quantum phases of bosons in a staggered flux lattice has been investigated as well [23,24].

In this paper, we study the interaction effects on the quantum phases of bosonic ${ }^{87} \mathrm{Rb}$ atoms in an optical lattice with magnetic flux that is staggered only along one direction, as was realized recently in Ref. [12]. While the noninteracting band structure is topologically trivial, still-interesting superfluid states emerge in the weak- as well as the strong-coupling limit. In particular, we show how two types of superfluid states in the weak-coupling limit evolve into the Mott-insulating regime through a tricritical point. Collective excitations in the Mott regime are studied in detail and give further evidence for the existence of a tricritical point. Multiple Dirac points are found in the collective excitation and we investigate the effects of interactions on the Dirac points and show that, while their dispersion depends strongly on interactions, their positions in the Brillouin zone remain intact in the Mott regime.

\section{SINGLE-PARTICLE PROPERTIES}

In the experiment in Ref. [12], the magnetic flux per plaquette with a magnitude $\frac{\pi}{2}$ that is periodic along the $\hat{y}$ direction, but staggered along the $\hat{x}$ direction, is realized with laser-assisted hopping in a superlattice; see Fig. 1(a). The magnitudes of the hopping amplitude along the $\hat{x}$ and $\hat{y}$ directions are given by $K$ and $J$, respectively. Due to the presence of the magnetic flux, the hopping Hamiltonian acquires Peierls phases and takes the form $H_{\text {hop }}=-\sum_{\mathbf{R}}\left(K e^{ \pm i \delta \mathbf{k} \cdot \mathbf{R}} c_{\mathbf{R}}^{\dagger} c_{\mathbf{R}+a \hat{x}}+\right.$ $J c_{\mathbf{R}}^{\dagger} c_{\mathbf{R}+a \hat{y}}+$ H.c.), where $\mathbf{R}=m a \hat{x}+n a \hat{y}$ are lattice sites and $a$ is the lattice constant and is set to units in the following. The \pm sign in the Peierls phases refers to the even and odd sites along the $\hat{x}$ direction; $m$ and $n$ are integers. By a simple gauge transformation for only the even sites along the $\hat{x}$ direction, $c_{2 m \hat{x}+n \hat{y}} \rightarrow e^{i 2 m \delta k_{x}+i n \delta k_{y}} a_{m n}$, and renaming the odd sites $a_{(2 m+1) \hat{x}+n \hat{y}} \rightarrow b_{m n}$, the space-dependent phase factors can be removed and one obtains a Hamiltonian with a unit cell that consists of two nonequivalent sites [12] [see Fig. 1(a)], which we label as site $A$ and site $B$ ( $m$ now labels the unit cell along the $\hat{x}$ direction). In terms of these new operators, the single-particle Hamiltonian takes the form

$$
\begin{aligned}
H_{\mathrm{hop}}= & -\sum_{m, n}\left(K a_{m, n}^{\dagger} b_{m, n}+J e^{i \delta k_{y}} a_{m, n}^{\dagger} a_{m, n+1}+\text { H.c. }\right) \\
& -\sum_{m, n}\left(K e^{i \delta k_{x}} b_{m, n}^{\dagger} a_{m+1, n}+J b_{m, n}^{\dagger} b_{m, n+1}+\text { H.c. }\right) .
\end{aligned}
$$

In momentum space, $H_{\text {hop }}=-\sum_{\mathbf{k}} \psi_{\mathbf{k}}^{\dagger} H(\mathbf{k}) \psi_{\mathbf{k}}$, where $H(\mathbf{k})=H_{0}(\mathbf{k}) \boldsymbol{I}+\mathbf{H}(\mathbf{k}) \cdot \boldsymbol{\sigma}, \quad$ with $\quad H_{0}=J\left[\cos \left(k_{y}+\right.\right.$ $\left.\left.\delta k_{y}\right)+\cos \left(k_{y}\right)\right], \quad H_{x}(\mathbf{k})=K\left[\cos k_{x}+\cos \left(k_{x}+\delta k_{x}\right)\right]$, $H_{y}(\mathbf{k})=K\left[\sin \left(k_{x}+\delta k_{x}\right)-\sin k_{x}\right], \quad$ and $\quad H_{z}(\mathbf{k})=$ $J\left[\cos \left(k_{y}+\delta k_{y}\right)-\cos k_{y}\right]$. Here $\sigma=\left(\sigma_{x}, \sigma_{y}, \sigma_{z}\right)$ are the Pauli matrices and $\psi_{\mathbf{k}}^{\dagger}=\left(a_{\mathbf{k}}^{\dagger}, b_{\mathbf{k}}^{\dagger}\right)$, and $\boldsymbol{I}$ is the $2 \times 2$ identity matrix. The $A, B$ sublattice constitutes a pseudospin half degree of freedom. The single-particle spectrum constitutes two branches that are given by $E_{ \pm}(\mathbf{k})=H_{0}(\mathbf{k}) \pm|\mathbf{H}(\mathbf{k})|$ with the corresponding pseudospin points either along or opposite the direction of $\mathbf{H}(\mathbf{k})$. In the following, we set $\delta k_{x}=\delta k_{y}=\frac{\pi}{2}$, as was the case in Ref. [12]. 

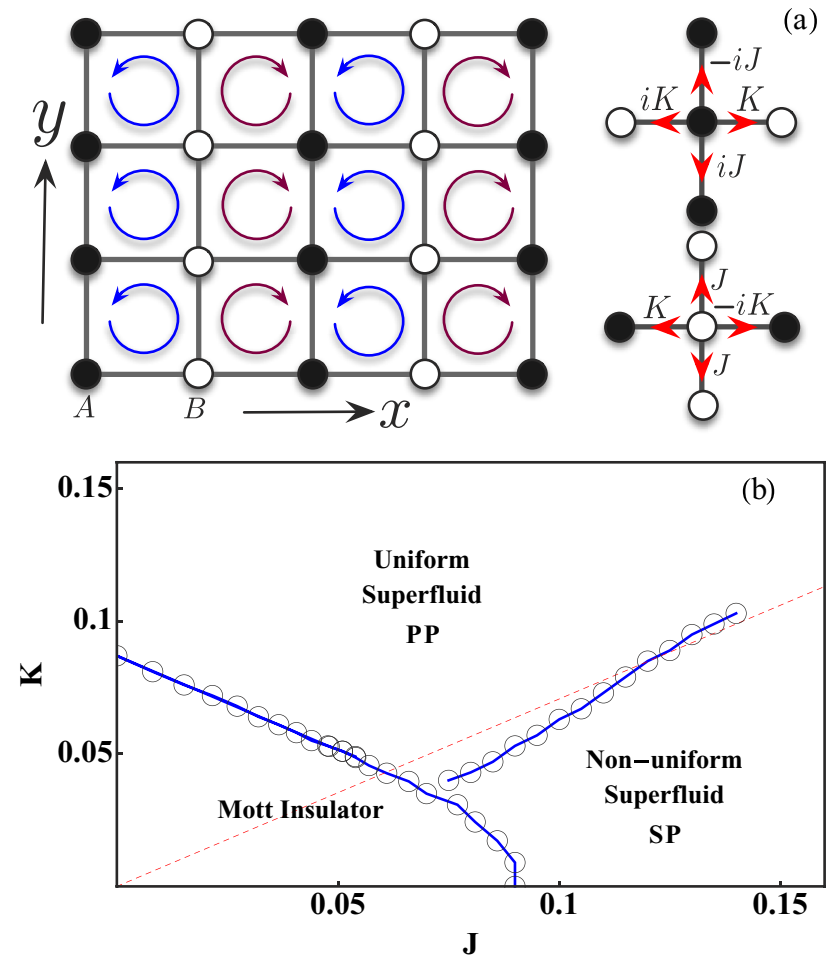

FIG. 1. (Color online) (a) Schematics of the two-dimensional square optical lattice with synthetic magnetic flux of magnitude $\frac{\pi}{2}$. The flux is periodic along the $\hat{y}$ direction, but staggered along the $x$ direction. This gives rise to a unit cell with two nonequivalent lattice sites $A$ (solid circle) and $B$ (open circle). The hopping amplitudes from $A$ and $B$ sites to their nearest neighbors are shown schematically. (b) Phase diagram based on the inhomogeneous mean-field theory for average filling factor $n_{A}+n_{B}=2$. Three phases are found within the inhomogeneous mean-field theory with cluster of dimension $8 \times 8$. Besides the Mott-insulating state, two superfluid states [plane-wave phase (PP) and stripe phase (SP)] are found and they terminate at a tricritical point. The dashed red line gives the boundary between PP and SP for a noninteracting gas. We have set $U$ as the energy unit.

The original Hamiltonian obeys the combined symmetry operations of time reversal $\mathcal{T}$ and spatial translation along the $\hat{x}$ direction by one unit of lattice constant, $T_{\hat{x}}$, namely $T_{\hat{x}}^{-1} \mathcal{T}^{-1} H_{\text {hop }} \mathcal{T} T_{\hat{x}}=H_{\text {hop }}$. With the transformed Hamiltonian Eq. (1), the spectrum exhibits the symmetry $E_{-}(\mathbf{k})=$ $-E_{+}(\mathbf{k}+\pi \hat{y})$. The lowest energy states depend on the ratio of $J / K$ [12]. For $J / K<\sqrt{2}$, the ground state is at $\left(q_{x}, q_{y}\right)=\left(-\frac{\pi}{4},-\frac{\pi}{4}\right)$, whereas for $J / K>\sqrt{2}$, there are two degenerate minima at $\left(-\frac{\pi}{4},-\frac{\pi}{2}+\frac{r}{2}\right)$ and $\left(-\frac{\pi}{4},-\frac{r}{2}\right)$, where $r=\arcsin \left[2(K / J)^{2}\right]$. There are also two nondegenerate Dirac points at $\frac{1}{2}\left(\pi-\delta k_{x},-\delta k_{y}\right)$ and $\frac{1}{2}\left(\pi-\delta k_{x}, 2 \pi-\delta k_{y}\right)$. By changing the angle between the two Raman beams, one can move the Dirac points around in the first Brillouin zone $\left[-\frac{\pi}{2}, \frac{\pi}{2}\right]_{\hat{x}} \otimes[-\pi, \pi]_{\hat{y}}$.

\section{MEAN-FIELD PHASE DIAGRAM}

The quantum phases of the model with general $\delta k_{x}=\delta k_{y}$ has been investigated extensively in the work by Möller and Cooper [24], where they have used the exact diagonalization technique for noninteger fillings and with isotropic hopping
$K=J$. They concluded that for any magnetic flux the ground state is always a condensate, irrespective of the interaction strength. The condensate, however, may exhibit spontaneous density modulations for general flux. In this work, we concentrate on integer filling and, in particular, we are interested in the transition from superfluid to Mott-insulating states. Let us thus assume the simplest form of interaction, as is appropriate to the optical lattice setting,

$$
H_{\text {int }}=\frac{U}{2}\left[n_{\mathrm{A}}\left(n_{\mathrm{A}}-1\right)+n_{\mathrm{B}}\left(n_{\mathrm{B}}-1\right)\right],
$$

where $U>0$ is the on-site repulsion.

\section{A. Weak-coupling superfluids}

Now, let us discuss the condensate structure in the weakinteracting limit, $J, K \gg U$. For $J / K<\sqrt{2}$, there is only a single ground state and the condensate wave function can be written as

$$
\left[\begin{array}{l}
\varphi_{\mathrm{A}} \\
\varphi_{\mathrm{B}}
\end{array}\right]_{\mathrm{PP}}=\sqrt{n} \frac{1}{\sqrt{2}}\left[\begin{array}{c}
1 \\
e^{i \frac{\pi}{4}}
\end{array}\right] e^{-i \frac{1}{4} \pi(2 m+n)},
$$

where $n$ is the average number per unit cell. The density is uniform and the phase of the condensate modulates along the $\hat{x}$ and $\hat{y}$ directions with period of eight lattice sites. We label this as the plane-wave phase (PP). On the other hand, when $J / K>\sqrt{2}$, there are two degenerate minima and, in the presence of interaction, a general ansatz for the ground-state wave function can be written as a superposition of two spinor wave functions at momenta $\left(-\frac{\pi}{4},-\frac{\pi}{4}+q\right)$ and $\left(-\frac{\pi}{4},-\frac{\pi}{4}-q\right)$, lying along the $\hat{y}$ direction, symmetric with respect to the point $\left(-\frac{\pi}{4},-\frac{\pi}{4}\right)[25]$ :

$$
\begin{aligned}
{\left[\begin{array}{c}
\varphi_{\mathrm{A}} \\
\varphi_{\mathrm{B}}
\end{array}\right]_{\mathrm{SP}}=} & \sqrt{n}\left[C_{1}\left[\begin{array}{c}
\sin \frac{\theta}{2} \\
\cos \frac{\theta}{2} e^{i \frac{\pi}{4}}
\end{array}\right] e^{-i \frac{1}{4} \pi(2 m+n)} e^{i q n}\right. \\
& \left.+C_{2}\left[\begin{array}{c}
\cos \frac{\theta}{2} e^{i \frac{3 \pi}{4}} \\
\sin \frac{\theta}{2}
\end{array}\right] e^{-i \frac{1}{4} \pi(2 m+n)} e^{-i q n}\right],
\end{aligned}
$$

where $q$ is a variational parameter that should be determined, together with $C_{1}$ and $C_{2}$, by minimizing the mean-field energy $E\left(q, C_{1}, C_{2}\right) \equiv\left\langle H_{\text {hop }}+H_{\text {int }}\right\rangle$. We note that if $q=0$, then ansatz Eq. (4) reduces to Eq. (3). For later convenience, we set $\left|C_{1}\right|=\cos \alpha$ and $\left|C_{2}\right|=\sin \alpha$; anticipating the relative phase between $C_{1}$ and $C_{2}$ is irrelevant for energy minimization, as it controls only the displacement of the stripe pattern. The parameter $\theta(q)$ characterizes the spin wave function at two degenerate minima and is given by $\cos \theta(q)=\frac{\sqrt{2} J \sin q}{\sqrt{2 J^{2} \sin ^{2} q+4 K^{2}}}$. The mean-field energy is then given by

$$
\begin{aligned}
E(q)= & -n \sqrt{2} J \cos q-n \sqrt{2 J^{2} \sin ^{2} q+4 K^{2}} \\
& +\frac{1}{8} n^{2}\left[3+\cos 4 \alpha-\frac{K^{2}(1+3 \cos 4 \alpha)}{2 K^{2}+J^{2} \sin ^{2} q}\right] .
\end{aligned}
$$

We need to minimize Eq. (5) for various values of $J$ and $K$. For $n=2$, it turns out that in general there are three possible phases: (i) a plane-wave phase with $q=0$ [Eq. (3)]; (ii) a stripe phase with $q \neq 0$ where, however, either $C_{1}$ or $C_{2}$ equals zero [Eq. (4)]; and (iii) a stripe phase with $q \neq 0$ and $\left|C_{1}\right|=$ $\left|C_{2}\right|=\frac{1}{\sqrt{2}}$ [Eq. (4)]. For relatively small values of $K$ and $J$ ( $K<3.2$ and $J<1.5$ ), only two phases, (i) and (iii), remain 
and they persist towards the Mott-superfluid transition, which is consistent with the inhomogeneous mean-field theory to be discussed below. According to Eq. (4), the density modulates along the $\hat{y}$ direction with form $\frac{n}{4} \sin \theta \cos (2 q n)$, while it stays uniform along the $\hat{x}$ direction. On the other hand, the phase of the condensate modulates with a period of 8 along the $\hat{x}$ direction while it varies in general noncommensurately along the $\hat{y}$ direction.

In the strong-coupling limit, $U \gg J, K$, the system enters the Mott-insulating regime with one boson per site and a finite excitation gap. Within Gutzwiller-type meanfield theory, the ground-state wave function takes the form $|\Psi\rangle=\prod_{m, n} a_{m, n}^{\dagger} b_{m, n}^{\dagger}|\mathrm{vac}\rangle$. As a result, there is no superfluid order: $\varphi_{\mathrm{A}}(m, n)=\varphi_{\mathrm{B}}(m, n)=0$, where $\varphi_{\mathrm{A}}(m, n)=\left\langle a_{m, n}\right\rangle$ and $\varphi_{\mathrm{B}}(m, n)=\left\langle b_{m, n}\right\rangle$ are the order parameters. Furthermore, the density is uniform. This is, however, no longer the case when the system enters into the superfluid states. In that case, it is possible for the system to develop both the superfluid and the density order and this is indeed what we find within mean-field theory.

\section{B. Mott-superfluid transition and the tricritical point}

To investigate how two types of condensate structures discussed above, (i) and (iii), evolve into the Mott state, we make use of the standard mean-field theory and decouple the hopping term as $a_{m, n}^{\dagger} b_{m, n}=\varphi_{\mathrm{A}}^{*}(m, n) b_{m, n}+$ $a_{m, n}^{\dagger} \varphi_{\mathrm{B}}(m, n)-\varphi_{\mathrm{A}}^{*}(m, n) \varphi_{\mathrm{B}}(m, n)+\widetilde{a}_{m, n}^{\dagger} \widetilde{b}_{m, n}$, and the fluctuation term $\widetilde{a}_{m, n}^{\dagger} \widetilde{b}_{m, n} \equiv\left[a_{m, n}^{\dagger}-\varphi_{\mathrm{A}}^{*}(m, n)\right]\left[b_{m, n}-\varphi_{\mathrm{B}}(m, n)\right]$ is neglected. With a similar decoupling scheme for other hopping terms, one obtains an effective single site Hamiltonian for the $A$ sublattice:

$$
\begin{aligned}
H_{\mathrm{MF}}^{(A)}= & \frac{U}{2}\left[n_{\mathrm{A}}\left(n_{\mathrm{A}}-1\right)\right]-\left[K \varphi_{\mathrm{B}}^{*}(m, n)+i K \varphi_{\mathrm{B}}^{*}(m-1, n)\right. \\
& \left.+i J \varphi_{\mathrm{A}}^{*}(m, n-1)-i J \varphi_{\mathrm{A}}^{*}(m, n+1)\right] a_{m, n}+\text { H.c. }
\end{aligned}
$$

Similarly, one can write $H_{\mathrm{MF}}^{(B)}$ for the $B$ sublattice. $H_{\mathrm{MF}}^{(A, B)}$ couples to its nearest neighbors through the mean fields $\varphi_{\mathrm{A}}(m, n)$ and $\varphi_{\mathrm{B}}(m, n)$ which are in general nonuniform in space and are determined self-consistently.

Unlike the standard Bose-Hubbard model where the ground state is expected to show translational symmetry, consistent with the underlying lattice, the ground state in our case may show modulations because of the complex and nonuniform hopping amplitudes along the $\hat{x}$ direction, as was shown in the weak-coupling analysis in Sec. III A. The superfluid-toMott-insulating transition, if it exists, is still going to be of the Kosterlitz-Thouless type, albeit with a nonuniform order parameter. As a result, we perform the mean-field calculation on a cluster of $N \times N$ square lattices. Assuming initial order parameters (in general, complex) on the $N \times N$ cluster, we calculate the new order parameter on a particular site based on the old order parameters at its neighboring sites. We iterate the calculation for each lattice site within the cluster until self-consistency for the entire cluster is achieved. A periodic boundary condition is used in the calculation. We perform our calculation with $N=8$ for the results presented in this paper.
The mean-field phase diagram is shown in Fig. 1(b) for an average of one particle per site, $n=2$, and one finds three phases. For small $K$ and $J$, the system is in the Mott-insulating state, while, depending on the ratio of $J / K$, the strong-coupling superfluid state exhibits two different phases. The PP, which occurs when the ratio $J / K$ is small, has uniform density while the phases modulate along the $\hat{x}$ and $\hat{y}$ directions with a period of 8 lattice sites; for a larger ratio $J / K$, the SP with density modulation along $\hat{y}$ occurs while the phases modulate along $\hat{x}$ with a period of 8 lattice sites. These features are all reminiscent of the weak-coupling superfluid phase and have been checked for larger cluster sizes [26]. The three phases meet at a tricritical point which, within mean-field theory, is at $J=0.07$ and $K=0.035$. At the tricritical point, two symmetries are broken simultaneously: $U(1)$ symmetry associated with the existence of a superfluid and a $Z_{2}$ symmetry associated with the existence of an Ising order parameter describing the existence of density modulations. We defer a detailed study of the tricritical point until later [27]. The fact that a single Mott state makes transitions to two different superfluid states can also be identified from the excitation spectrum of the Mott state. It is expected that the emerging superfluid states will inherit the structure of the softened collective mode from the Mott-insulating state that depends on the ratio $J / K$. This can serve as further evidence for the existence of a tricritical point.

\section{COLLECTIVE EXCITATIONS}

Having established the mean-field phase diagram, let us now discuss the collective excitations in the Mott-insulating phase. Since density is uniform in the Mott regime (one particle per site), the unit cell turns out to be composed of $A$ and $B$ sites, as in the noninteracting case. Let us then define the local basis for the mean-field Hamiltonian $H_{\mathrm{MF}}^{(\chi)}$ $(\chi=A, B)$ as $|\chi ; \alpha\rangle$, with eigenenergy $E_{\alpha}^{\chi}$. If we now define the standard basis operators $L_{\alpha \alpha^{\prime}}^{\chi}=|\chi, \alpha\rangle\left\langle\chi, \alpha^{\prime}\right|$, where $\alpha, \alpha^{\prime}$ label the eigenstates, then $H_{\mathrm{MF}}^{(\chi)}=\sum_{\alpha} E_{\alpha}^{\chi} L_{\alpha \alpha}^{\chi}$, diagonal in the basis $|\chi, \alpha\rangle$. On the other hand, the fluctuation terms that have been neglected in the mean-field treatment can now be written in terms of $L_{\alpha \alpha^{\prime}}^{\chi}: \widetilde{a}_{m, n}^{\dagger} \widetilde{b}_{m, n}=\sum_{\alpha \alpha^{\prime} ; \beta \beta^{\prime}} D_{\alpha \alpha^{\prime}}^{+A} D_{\beta \beta^{\prime}}^{-B} L_{\alpha \alpha^{\prime}}^{A} L_{\beta \beta^{\prime}}^{B}$ and $D_{\alpha \alpha^{\prime}}^{\sigma \chi}=\left\langle\chi, \alpha\left|\psi_{\chi}^{\sigma}-\left\langle\psi_{\chi}^{\sigma}\right\rangle\right| \chi, \alpha^{\prime}\right\rangle$, where $\sigma= \pm$ in the superscript denotes creation and annihilation operators and $\psi_{A}^{\sigma}=$ $a^{\sigma}$ and $\psi_{B}^{\sigma}=b^{\sigma}$. The full original Hamiltonian can now be written in terms of the operators $L_{\alpha \alpha^{\prime}}^{\chi}$.

To obtain the excitation spectrum, we define the single-particle Green function $\boldsymbol{G}_{\chi \chi^{\prime}}^{\sigma \sigma^{\prime}}\left(\mathbf{r}, \mathbf{r}^{\prime} ; t\right)=$ $-i\left\langle\mathcal{T} \psi_{\chi}^{\sigma}(\mathbf{r}, t) \psi_{\chi^{\prime}}^{\sigma^{\prime}}\left(\mathbf{r}^{\prime}, 0\right)\right\rangle$, where $\mathcal{T}$ is the time-ordering operator. Writing $\boldsymbol{G}_{\chi \chi^{\prime}}^{\sigma \sigma^{\prime}}$ in terms of standard basis operators and making use of the random phase approximation [28,29], we find the following equation for the Green function $\boldsymbol{G}_{\chi \chi^{\prime}}^{\sigma \sigma^{\prime}}$ (after Fourier transforming to the frequency-momentum space):

$$
\begin{aligned}
\boldsymbol{G}_{\chi \chi^{\prime}}^{\sigma \sigma^{\prime}}(\mathbf{q}, \omega)= & \frac{1}{2 \pi} \delta_{\chi \chi^{\prime}} \boldsymbol{B}_{\chi}^{\sigma \sigma^{\prime}}(\omega)-2 \sum_{i} e^{i \mathbf{q} \cdot \delta_{i}}\left[\boldsymbol{B}_{\chi}^{\sigma-}(\omega) t_{\delta_{i}}^{\chi} \boldsymbol{G}_{\chi_{i} \chi^{\prime}}^{+\sigma^{\prime}}\right. \\
& \left.\times(\mathbf{q}, \omega)+\boldsymbol{B}_{\chi}^{\sigma+}(\omega) t_{\delta_{i}}^{\chi *} \boldsymbol{G}_{\chi_{i} \chi^{\prime}}^{-\sigma^{\prime}}(\mathbf{q}, \omega)\right]
\end{aligned}
$$


in which $\boldsymbol{\delta}_{i}$ 's are the four unit vectors which point outwards from a particular site; $t_{\delta_{i}}^{\chi}$ gives the hopping amplitude from a site with sublattice index $\chi$, along the direction $\delta_{i}$, to its neighboring site with sublattice index $\chi_{i}$ [see Fig. 1(a)]. The function $\boldsymbol{B}_{\chi}^{\sigma \sigma^{\prime}}(\omega)$ is given by

$$
\boldsymbol{B}_{\chi}^{\sigma \sigma^{\prime}}(\omega)=\sum_{\delta \delta^{\prime}} \frac{\left\langle L_{\delta \delta}^{\chi}\right\rangle-\left\langle L_{\delta^{\prime} \delta^{\prime}}^{\chi}\right\rangle}{\omega+E_{\delta}^{\chi}-E_{\delta^{\prime}}^{\chi}} D_{\delta \delta^{\prime}}^{\sigma \chi} D_{\delta^{\prime} \delta}^{\sigma^{\prime} \chi},
$$

where the average is taken over the mean-field ground state. The excitation spectrum is determined implicitly by setting $\operatorname{det}\left[\boldsymbol{G}^{-1}(\omega, \mathbf{k})\right]=0$.

In the Mott regime, there is no superfluid order and the situation simplifies considerably. Equation (7) becomes block diagonal with $\boldsymbol{G}^{++}=\boldsymbol{G}^{--}=0$ and we have $(1+\mathcal{B}) \boldsymbol{G}^{-+}=$ $\boldsymbol{J}$, where explicitly

$$
\mathcal{B}=\left[\begin{array}{cc}
-2 J \sin q_{y} \boldsymbol{B}_{A}^{-+} & K\left(e^{i q_{x}}-i e^{-i q_{x}}\right) \boldsymbol{B}_{A}^{-+} \\
K\left(i e^{i q_{x}}+e^{-i q_{x}}\right) \boldsymbol{B}_{B}^{-+} & 2 K \cos q_{y} \boldsymbol{B}_{B}^{-+}
\end{array}\right]
$$

and the matrix $\boldsymbol{J}$ is given by

$$
\boldsymbol{J}=\left[\begin{array}{cc}
\boldsymbol{B}_{A}^{-+} & 0 \\
0 & \boldsymbol{B}_{B}^{-+}
\end{array}\right] .
$$

As a result, the excitation spectrum is determined by $\operatorname{det}(1+$ $\mathcal{B})=0$ and we denote it as $E(\mathbf{k})$. There is also a similar branch for the Green function $\boldsymbol{G}^{+-}$, whose solution is given by $E^{\prime}(\mathbf{k})$. We note the following relation $E(\mathbf{k})=-E^{\prime}(-\mathbf{k})$ and concentrate on $E(\mathbf{k})$ below.

In Fig. 2, we show the two branches of quasihole excitations closest to zero energy. One particular feature that is worth
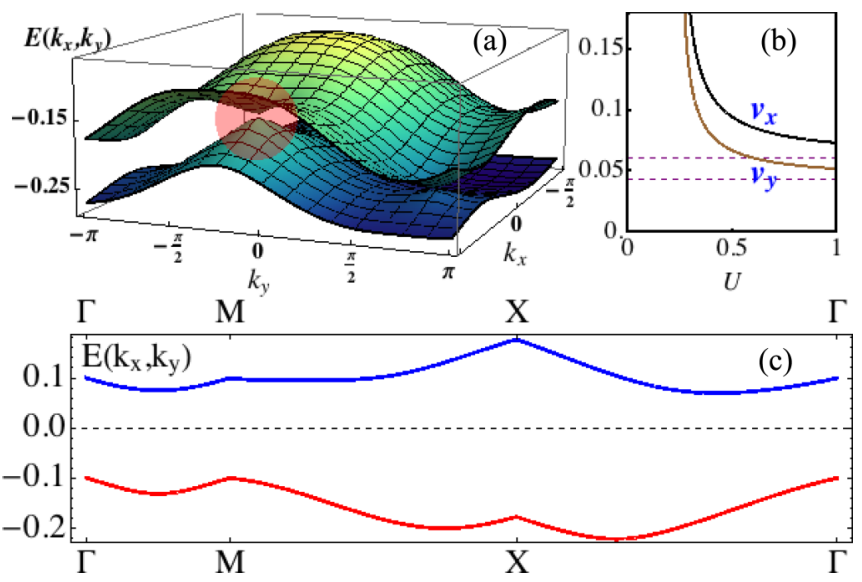

FIG. 2. (Color online) (a) Quasihole excitation for the two bands closest to zero energy. There are two Dirac points in the spectrum at $\mathbf{k}_{1}=\left(\frac{\pi}{4}, \frac{3 \pi}{4}\right)$ and $\mathbf{k}_{2}=\left(\frac{\pi}{4},-\frac{\pi}{4}\right)$. The shaded region shows the Dirac cone at $\mathbf{k}_{2}$. (b) The velocity of the quasihole along the $k_{x}$ and $k_{y}$ directions for the Dirac point $\mathbf{k}_{2}$, as a function of $U$ in the Mott regime. The dashed line corresponds to the noninteracting value and has a ratio $\sqrt{2}$. (c) Quasiparticle and quasihole bands closest to zero energy in units of $U$. In the Mott regime, a finite gap exists between the quasiparticle and quasihole bands. The gap decreases as one approaches the superfluid phases. There is no particle-hole symmetry in the excitation spectrum. For $(\mathrm{a}-\mathrm{c}), K=J=0.03$. The average number of bosons per site is set to one. noting is the appearance of Dirac points in the excitation spectrum at the positions corresponding to the noninteracting case or their symmetry-related points [see Fig. 2(a)]. The dispersion close to the Dirac point is linear and can be characterized by two velocities $v_{x, y}$ along $k_{x, y}$, respectively. While the positions of the Dirac points are unaffected by the strong interactions, its dispersion is significantly renormalized from the noninteracting values, as shown in Fig. 2(b). In the deep Mott regime $U \gg J, K$, quasiparticles are essentially doublon and hole [30], which hop with a phase relation that is the same as in the noninteracting case, apart from the renormalization of the amplitudes and an overall energy shift. As one approaches the Mott-superfluid transition boundary, the Dirac cone becomes sharper. Surprisingly the "anisotropy" of the Dirac cone, $\frac{v_{x}}{v_{y}}=\sqrt{2}$, remains the same as in the noninteracting case. In Fig. 2(c), we plot the quasiparticle and quasihole excitation closest to zero energy along a representative path in the first Brillouin zone, connecting symmetry points $\Gamma=(0,0), M=$ $\left(\frac{\pi}{2}, 0\right)$, and $X=\left(\frac{\pi}{2}, \pi\right)$. As expected, a finite gap always exists in the Mott-insulating regime and there is no particle-hole symmetry.

The above features can be understood from the structure of the Green function $\boldsymbol{G}^{-+}$. Its inverse can be written as
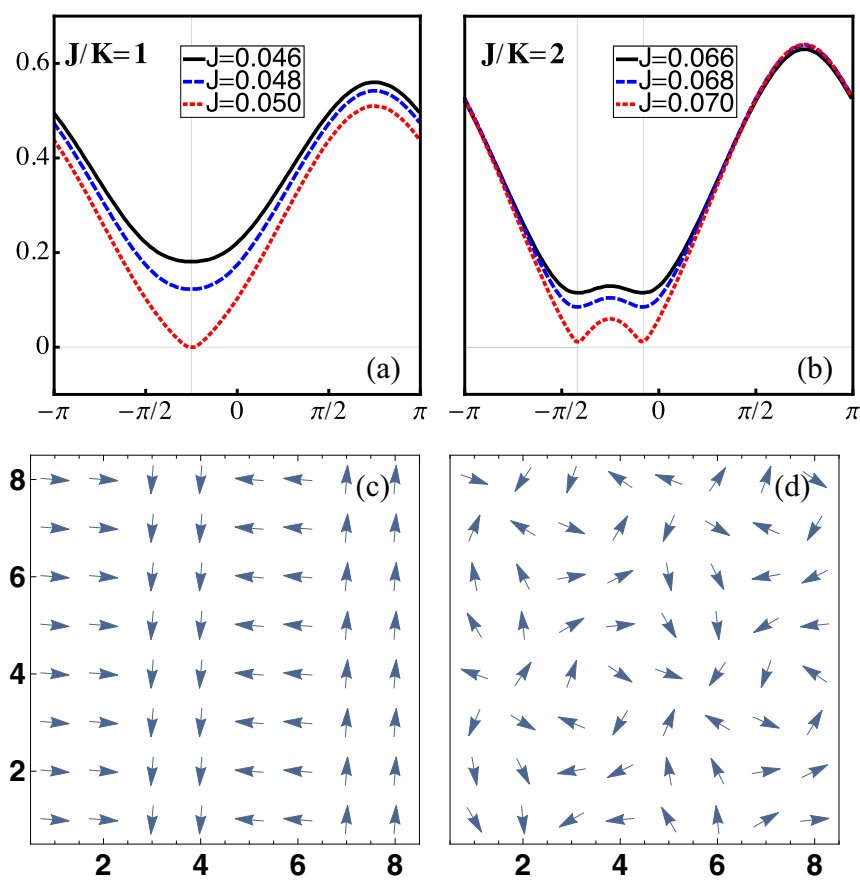

FIG. 3. (Color online) Softening of the excitation spectrums in Mott-insulating state as a function of $k_{y}$, for $k_{x}=-\pi / 4$, along two values of $J / K$, making the transition to PP and SP. (a) $J / K=1$, and the excitation spectrum goes to zero at $k_{x}=-\pi / 4, k_{y}=-\pi / 4$, consistent with the structure of the strong-coupling superfluid state. (b) $J / K=2$, and the excitation spectrum goes to zero at two values of $k_{y}$, indicating the emergence of condensate with two momentum components. (c, d) The phases (directions of the arrows) of superfluid order parameters at each site for PP and SP, respectively. Overall $\pi / 4$ modulation along $y$ has been removed to make the comparison between (c) and (d) clear. We have set $U$ as the energy unit. 
$\left(\boldsymbol{G}^{-+}\right)^{-1}=\boldsymbol{J}^{-1}(1+\mathcal{B}) \equiv h_{0}+\boldsymbol{h} \cdot \boldsymbol{\sigma}$. In the case we are considering, namely one boson per site, $\boldsymbol{B}_{A}^{-+}=\boldsymbol{B}_{B}^{-+}$and we find $h_{x}=H_{x}, h_{y}=H_{y}$, and $h_{z}=H_{z}$, the same as in the noninteracting case. With this, it is straightforward to conclude that the Dirac points will remain at their original positions and, in addition, the quasiparticle excitations have the same spinor wave functions as that of a single particle.

Finally, let us discuss the behavior of collective excitations close to the Mott-superfluids transition and show how it is connected to the emergent superfluid states. Figure 3(a) shows that for fixed ratio $J / K=1$, the excitation energy approaches zero at momentum $\left(-\frac{\pi}{4},-\frac{\pi}{4}\right)$, which corresponds to the modulations of the plane-wave phase. On the other hand, for fixed ratio $J / K=2$ [see Fig. 3(b)], the excitation energy approaches zero at $t w o$ distinct $\mathbf{k}$ points $\left(-\frac{\pi}{4},-\frac{\pi}{4}+q\right)$ and $\left(-\frac{\pi}{4},-\frac{\pi}{4}-q\right)$, where $q$ depends on the values $J$ and $K$, as well as interaction $U$. This suggests that the emergent condensate is of the form of Eq. (4). In Figs. 3(c) and 3(d), a clear difference between the phase modulations in the PP and SP states is shown with the overall $\exp \left(-i \frac{\pi}{4} n\right)$ factor removed for clear comparison.

\section{CONCLUSION}

We have shown how weak-coupling superfluid states evolve into the Mott-insulating state in a Bose-Hubbard model with synthetic staggered flux. It is predicted that a tricritical point exists where the plane-wave state, the striped state, and the Mott-insulating state terminate. While excitations in the Mott regime give further supporting evidence of the existence of a tricritical point, further studies are necessary to illustrate its nature. The effects of interaction on topological Dirac points are quantified and discussed.

\section{ACKNOWLEDGMENTS}

We thank Tin-Lun Ho, Yun Li, Zhenhua Yu, and Hui Zhai for valuable discussions and suggestions. Y.J. acknowledges support from the University of Hong Kong postgraduate fellowship and postgraduate scholarship. This work is partially supported by a grant from the Research Grants Council of the Hong Kong Special Administrative Region, China (Grant No. HKU 709313P) and a startup grant from the University of Hong Kong.
[1] R. E. Prange and S. M. Girvin, The Quantum Hall Effect (Springer-Verlag, Berlin, 1987).

[2] X.-L. Qi and S.-C. Zhang, Rev. Mod. Phys. 83, 1057 (2011).

[3] M. Z. Hasan and L. C. Kane, Rev. Mod. Phys. 82, 3045 (2010).

[4] Y. J. Lin, R. L. Compton, A. R. Perry, W. D. Phillips, J. V. Porto, and I. B. Spielman, Phys. Rev. Lett. 102, 130401 (2009).

[5] Y.-J. Lin, R. L. Compton, K. Jiménez-García, J. V. Porto, and I. B. Spielman, Nature (London) 462, 628 (2009).

[6] Y.-J. Lin, K. Jiménez-García, and I. B. Spielman, Nature (London) 471, 83 (2011).

[7] L. W. Cheuk, A. T. Sommer, Z. Hadzibabic, T. Yefsah, W. S. Bakr, and M. W. Zwierlein, Phys. Rev. Lett. 109, 095302 (2012).

[8] P. Wang, Z. Q. Yu, Z. Fu, J. Miao, L. Huang, S. Chai, H. Zhai, and J. Zhang, Phys. Rev. Lett. 109, 095301 (2012).

[9] J. Y. Zhang, S. C. Ji, Z. Chen, L. Zhang, Z. D. Du, B. Yan, G. S. Pan, B. Zhao, Y. J. Deng, H. Zhai, S. Chen, and J. W. Pan, Phys. Rev. Lett. 109, 115301 (2012).

[10] J. Struck, C. Oelschlaeger, R. Le Targat, P. Soltan-Panahi, A. Eckardt, M. Lewenstein, P. Windpassinger, and K. Sengstock, Science 333, 996 (2011).

[11] J. Struck, C. Olschlager, M. Weinberg, P. Hauke, J. Simonet, A. Eckardt, M. Lewenstein, K. Sengstock, and P. Windpassinger, Phys. Rev. Lett. 108, 225304 (2012).

[12] M. Aidelsburger, M. Atala, S. Nascimbène, S. Trotzky, Y. A. Chen, and I. Bloch, Phys. Rev. Lett. 107, 255301 (2011).

[13] M. Aidelsburger, M. Atala, M. Lohse, J. T. Barreiro, B. Paredes, and I. Bloch, Phys. Rev. Lett. 111, 185301 (2013).

[14] H. Miyake, G. A. Siviloglou, C. J. Kennedy, W. C. Burton, and W. Ketterle, Phys. Rev. Lett. 111, 185302 (2013).
[15] P. G. Harper, Proc. Phys. Soc. London Sect. A 68, 874 (1955).

[16] D. R. Hofstadter, Phys. Rev. B 14, 2239 (1976).

[17] Y. M. Lu and A. Vishwanath, Phys. Rev. B 86, 125119 (2012).

[18] X. Chen, Z. C. Gu, Z. X. Liu, and X. G. Wen, Phys. Rev. B 87, 155114 (2013).

[19] T. Senthil and Michael Levin, Phys. Rev. Lett. 110, 046801 (2013).

[20] M. A. Metlitski, C. L. Kane, and M. P. A. Fisher, Phys. Rev. B 88, 035131 (2013).

[21] C. Xu and T. Senthil, Phys. Rev. B 87, 174412 (2013).

[22] S. Powell, R. Barnett, R. Sensarma, and S. Das Sarma, Phys. Rev. Lett. 104, 255303 (2010).

[23] L.-K. Lim, C. M. Smith, and A. Hemmerich, Phys. Rev. Lett. 100, 130402 (2008).

[24] G. Möller and N. R. Cooper, Phys. Rev. A 82, 063625 (2010).

[25] Y. Li, L. P. Pitaevskii, and S. Stringari, Phys. Rev. Lett. 108, 225301 (2012).

[26] Larger cluster sizes with 16 sites along the $\hat{x}$ direction and a variable number of sites along the $\hat{y}$ direction, from 8 to 100 , have been used to explore the phases, with similar conclusions; in particular, the existence of a tricritical point is hardly modified.

[27] Juan Yao and Shizhong Zhang (unpublished).

[28] K. Sheshadri, H. Krishbanurthy, R. Pandit, and T. Ramakrishnan, Europhys. Lett. 22, 257 (1993).

[29] Y. Ohashi, M. Kitaura, and H. Matsumoto, Phys. Rev. A 73, 033617 (2006).

[30] A. L. Chudnovskiy, D. M. Gangardt, and A. Kamenev, Phys. Rev. Lett. 108, 085302 (2012). 